\title{
CORRECTIONS TO STAR CATALOGUES FROM SATELLITE OBSERVATIONS
}

\author{
CAROL A. WILLIAMS \\ University of South Florida, Florida, U.S.A.
}

\begin{abstract}
A theoretical investigation is made to explore the possibility of obtaining corrections to star catalogues from the observations of artificial satellites. A model is established to represent the system formed when such corrections are determined simultaneously with corrections to the geocentric position of the observer. The covariance matrix is established analytically for the two-dimensional case. The resulting correlations and weights are discussed. Since this method presents an independent way of obtaining the systematic errors in star catalogues, it is related to the measurement of all quantities which depend on star positions.
\end{abstract}

Eichhorn (1970) made the first formal proposal to obtain the systematic errors of star catalogues from a reduction of the observations of artificial satellites. Since many of the quantities currently being obtained from satellite observations are of the same order of magnitude as the errors of the catalogues, it may be that such corrections can be obtained. The scheme requires that the orbit and the position of the observer be known. Since these are not usually known, it is necessary to see if correlations between all the unknown parameters and the expected mean errors with which they are determined are low enough to make a least squares adjustment practical.

The covariance matrix of a least squares adjustment can be found analytically in some cases by methods established by Eichhorn and Williams (1963). These methods are applied to a least squares system of a two dimensional problem. The unknown quantities are coefficients of the Fourier series representations of the errors in right ascension, $\Delta \alpha$, observer's longitude, $\Delta \lambda$, and his relative distance from the center of the Earth, $\Delta b / b_{\oplus}$. The elements of the covariance matrix are found as functions of two parameters: $\beta$, the reciprocal radius of an assumed circular orbit of the satellite in units of Earth radii, and $\zeta_{m}$, the maximum zenith distance over which observations at a single station are made. Furthermore, two least squares adjustments are considered; one in which only the right ascension, $\alpha$, is observed and one in which $\alpha$ and the topocentric distance of the satellite, $\varrho$, are both observed.

The only correlations that exist are between the like harmonics (e.g. sine-sine) of $\Delta \alpha$ and $\Delta \lambda$, and between opposite harmonics (sine-cosine) of $\Delta \lambda$ and $\Delta b / b_{\oplus}$ and of $\Delta \alpha$ and $\Delta b / b_{\oplus}$. The diagonal terms of the covariance matrix indicate that $\Delta \alpha$ is determined better for more distant satellites while $\Delta \lambda$ is better determined for closer ones. This is related to the fact that the parallax angle of the satellite decreases with increasing distance, making right ascensions more sharp. For the same parallax the reverse is true at lower altitudes. The correlations between $\Delta \alpha$ and $\Delta \lambda$ reflect the same phenomenon. For satellites much lower than $3000 \mathrm{~km}$ altitude, the correlations are so high as to made the solution impractical. 
In general the results for $\alpha$ and $\lambda$ improve as the range of zenith distance grows. $\Delta \alpha$ is fairly insensitive to the measurement of $\varrho$, but there is significant improvement of the results for $\Delta \lambda$ if $\varrho$ is observed.

For the standard errors of $\Delta b / b_{\oplus}$, the most important result is that the observer's distance from the center of the Earth cannot be found with much accuracy without distance determinations. The one exception is for observations of satellites closer than $3000 \mathrm{~km}$.

For high altitude satellites, for which distances are observed, there are very low correlations between the harmonics of $\Delta \alpha$ and $\Delta b / b_{\oplus}$. There are no correlations between the constant terms. The correlations between $\Delta \lambda$ and $\Delta b / b_{\oplus}$ are low for most cases and are not strongly influenced by the observation of $\varrho$. There is no correlation between the constant terms.

The analytical values given here for elements of the covariance matrix can be used to determine what type of satellite orbit should be chosen in order to correct the right ascensions or the geocentric position of an observer on the equator. The results do indicate that star catalogues can be corrected by an analysis of satellite positions, including data for the Moon. It seems possible to separate these corrections from corrections to the observer's location. The study indicates to what extent observations of the topocentric distance should be included along with observations of $\alpha$.

A further study is being made which incorporates variations in the standard deviations for $\varrho$ and $\alpha$. The results which incorporate observations of topocentric distances are the only ones affected. A major investigation of the three dimensional case is now being done.

\section{References}

Eichhorn, H.: 1970, Cospar Transactions, No. 7, p. 1.

Eichhorn, H. and Williams, C. A.: 1963, Astron. J. 68, 221.

\section{DISCUSSION}

$W$. Fricke: This investigation on the possibility of obtaining corrections to star catalogues from observations of artificial satellites is certainly very useful, in particular for the determination of regional systematic errors in $\alpha$ and $\delta$, if an accuracy of about \pm 0 " 1 could be at tained. Are you of the opnion that this will possible in view of the limitations in the accuracy of measurements on photographic plates?

C. A. Williams: The theoretical investigation shows that the variance of a harmonic coefficient in the Fourier expansion of $\Delta \alpha$ ranges from $0.01 / N$ to $0.6 / N$, where $N$ is the number of observations per radian. Some experiments we have made indicate that the theoretical method gives results which differ from an actual problem by $10 \%$ for a uniform distribution of observations. The agreement between theory and actual adjustments improves as $N$ increases. The theory predicts that we can obtain the harmonic coefficients with a mean error of $0.1 \sigma_{\alpha} / \sqrt{N}$ to $0.8 \sigma_{\alpha} / \sqrt{N}$, where $\sigma_{\alpha}$ is the standard error of the observations of $\alpha$. This range of values is approximately what is required, especially, if optimum values of the parameters, $\beta$ and $\zeta_{M}$, are chosen. 